\title{
Beating type 2 diabetes into remission
}

\section{Recognising and accurately coding reversal of type 2 diabetes is key to improving outcomes and reducing healthcare costs, argue Louise McCombie and colleagues}

\author{
Louise McCombie researcher ${ }^{1}$, Wilma Leslie researcher ${ }^{1}$, Roy Taylor professor ${ }^{2}$, Brian Kennon ${ }^{3}$, \\ Naveed Sattar professor ${ }^{4}$, Mike E J Lean professor ${ }^{1}$
}

\begin{abstract}
${ }^{1}$ Human Nutrition Section, School of Medicine, University of Glasgow, Glasgow, G31 2ER, UK; ${ }^{2}$ Magnetic Resonance Centre, Campus for Ageing and Vitality, University of Newcastle, Newcastle Upon Tyne, UK; ${ }^{3}$ Diabetes Centre, Queen Elizabeth University Hospital, Glasgow; ${ }^{4}$ University of Glasgow Biomedical Research Centre, Glasgow
\end{abstract}

Type 2 diabetes, generally perceived as progressive and incurable, now affects $5-10 \%$ of the population, about 3.2 million people in the UK. ${ }^{1}$ Until complications develop, most patients are managed entirely within primary care, with diabetes comprising a major part of general practice activity. About $10 \%$ of total UK NHS expenditure goes on treating diabetes, and international figures suggest that medical costs for people with diabetes are twofold to threefold greater than the average for age and sex matched people without diabetes.'

Application of current clinical guidelines to reduce glycated haemoglobin $\left(\mathrm{HbA}_{1 c}\right)$ levels and cardiovascular risks, primarily with drugs and generic lifestyle advice, has improved clinical outcomes, but many patients still develop vascular complications, and life expectancy remains up to six years shorter than in people without diabetes. ${ }^{2}$ The diagnosis carries important social and financial penalties for individuals, as well as poor health prospects.

Remission of diabetes (no longer having diabetes, at least for a period) is clearly attainable for some, possibly many, patients but is currently very rarely achieved or recorded. Greater awareness, documentation, and surveillance of remissions should improve health outcomes and reduce healthcare costs.

\section{Changing management}

In keeping with trends in most medical specialties, diabetes management is beginning to focus on reversible underlying disease mechanisms rather than treating symptoms and subsequent multisystem pathological consequences. ${ }^{34}$ Both (epi)genetic predisposition and ageing have a role in type 2 diabetes, but it is rare without weight gain.

Lowering blood glucose or $\mathrm{HbA}_{1 \mathrm{c}}$ concentrations remains the primary aim of management, as reflected in current clinical guidelines and the actions of licensed drugs. However, management and guidelines focus on use of antidiabetes drugs, with only lip service paid to diet and lifestyle advice. The scale of the market for drug therapies, and their likely inadequacy, is illustrated by the fact that 488 drugs (excluding insulins) are currently licensed worldwide to treat type 2 diabetes, with 70 generic compounds. ${ }^{5}$ They all lower blood glucose and $\mathrm{HbA}$ significantly, but no trials have yet examined drugs administered together with optimal diet and lifestyle advice for weight control.

In contrast, consistent evidence shows that weight loss is associated with extended life expectancy for people with diabetes, and that weight loss of around $15 \mathrm{~kg}$ often produces total biochemical remission of type 2 diabetes, restoring beta cell function. ${ }^{67}$ Recognition that accumulation of ectopic fat in the liver and pancreas impairs organ function to cause type 2 diabetes, but is reversible, has raised awareness that remission is possible. ${ }^{7}$ Media attention has encouraged increasing numbers of people with type 2 diabetes to lose weight and shed the diagnosis. Remission produces a strong sense of personal achievement and empowerment; it also benefits medical systems because patients no longer require antidiabetes drugs. The UK NHS currently spends almost $£ 1$ bn ( $€ 1$ bn; $\$ 1.3$ bn) a year $(£ 22 \mathrm{~m}$ a day) on antidiabetes drugs, and costs are rising worldwide as diabetes rates and drug prices escalate.

\section{Criteria for remission of diabetes}

There is no consensus on the criteria for remission of diabetes. The published criteria, and thus reported remission rates, vary, but all require being below the World Health Organization/American Diabetes Association (ADA) diagnostic thresholds for diabetes (table $1 \Downarrow$ ). Most recent publications, including the large Look AHEAD trial of lifestyle interventions ${ }^{10}$ refer to an ADA consensus group statement ${ }^{8}$ that defined test results below the diagnostic threshold for diabetes as partial remission and remission of "pre-diabetes" as complete remission.

However, using the term partial remission does not fully portray the logic or importance of becoming non-diabetic to patients. Although remission of prediabetes is desirable because it reduces 
cardiovascular risk, that routine management should aim to help patients escape the diagnostic category of diabetes, with its personal burdens and risks of microvascular disease. That requires using the $\mathrm{WHO}$ diagnostic thresholds for diabetes to establish remission and accepting that some patients will continue to have impaired glucose tolerance or prediabetic fasting glucose and $\mathrm{HbA}$, profiles. $^{8}$ Measurement of either $\mathrm{HbA}_{1 \mathrm{c}}$ or blood glucose is sufficient to identify remission. To avoid misclassification resulting from measurement error or biological variation, we recommend that patients should have two tests results (usually $\mathrm{HbA}_{1 \mathrm{c}}$ ) below the diagnostic threshold, at least two months apart to confirm remission. This reflects about four months of normoglycaemia. Patients in remission should be kept under regular review with annual testing (table $1 \Downarrow)$.

\section{Potential metabolic value of remission}

Clinical outcomes for people with type 2 diabetes are all worse at higher glucose or $\mathrm{HbA}_{1 \mathrm{c}}$ concentrations. ${ }^{10}$ No study has yet reported outcomes for people who achieve remission, but good glycaemic control through drug treatment improves microvascular outcomes (retinopathy, neuropathy, nephropathy) in both type 1 and type 2 diabetes. ${ }^{11}$ Reduced cardiac events have also been observed in patients with type 2 diabetes after bariatric surgery. ${ }^{12}$ In contrast, some drug trials have indicated increased mortality when $\mathrm{HbA}_{1 \mathrm{c}}$ approaches normal levels, probably through hypoglycaemia provoking arrhythmias. ${ }^{13}$ Look AHEAD, a trial of lifestyle intervention in patients with mean diabetes duration of five years, reported $11.5 \%$ remission at 12 months with $8.6 \%$ weight loss, but that proportion fell by about $30 \%$ annually. ${ }^{10}$ Health outcomes of participants have not yet been reported, but the limited evidence and first principles suggest that remission of diabetes (without antidiabetes drugs) should enhance overall prognosis, becoming a primary management target as early as possible after diagnosis. ${ }^{10}$

\section{Coding for health records}

Remission of type 2 diabetes can be inferred from routine records of patients labelled diabetic who have non-diabetic biochemical test results (usually $\mathrm{HbA}_{1 \mathrm{c}}<48 \mathrm{mmol} / \mathrm{mol}$ ), when there is no record that antidiabetes drugs are prescribed. ${ }^{14}$ In the UK, clinicians have used Read codes to record patients' findings and procedures since $1985^{15}$ but are now converting to the international SNOMED system. The basic coding framework will remain, however.

The Read code dictionary includes codes 21263 or $212 \mathrm{H}$ for "diabetes resolved" and C10P for "diabetes in remission." Diabetes resolved is used for patients misdiagnosed with diabetes or in whom diabetes was secondary to a factor that has since been removed, such as withdrawal of steroid treatment. Such patients do not require annual reviews or surveillance. Code C10P should be used for patients who have achieved remission of type 2 diabetes, usually by substantial weight loss. These patients may be considered non-diabetic for matters such as insurance, driving, or employment but as the code is diagnostic they will remain scheduled for annual reviews and retinal screening programmes.

\section{Achieving remission}

In the few countries with data, patients are seldom recorded as having diabetes in remission. Karter and colleagues found remissions in only $0.14 \%$ of 120000 US patients followed for seven years. ${ }^{14}$ The Scottish Care Information Diabetes database, which includes every patient in Scotland, shows that less than $0.1 \%$ of those with type 2 diabetes (245/254 208) were coded as being in remission in March 2017 (table $2 \Downarrow$ ).

Lack of agreed criteria and guidance over recoding may have led to hesitation in coding remission, but the main reason for the low recording is probably that few patients are attempting or achieving remission. Patients and doctors may be unaware that type 2 diabetes can be reversed, despite recent publicity. The feasibility of sustained substantial intentional dietary weight loss is widely doubted, despite publications of structured approaches using an initial formula diet replacement to achieve rapid substantial weight loss and then maintain $12-15 \mathrm{~kg}$ loss at 12 months and beyond. ${ }^{16} 17$

The 2010 guideline of the Scottish Intercollegiate Guidelines Network recommends sustained weight loss above $10 \%$ of body weight or $15 \mathrm{~kg}$ for people with severe and complicated obesity, including type 2 diabetes. ${ }^{18}$ This is difficult to achieve, even with bariatric surgery, and only $75-80 \%$ of patients who succeed are rewarded with diabetes remission. ${ }^{9}$

The drivers and barriers to successful remission are not fully evaluated but are likely to include age, body mass index, duration of diabetes, $\mathrm{HbA}_{1 \mathrm{c}}$ concentrations, and drug treatment. ${ }^{10}$ Physical and social environments, emotional states, and self regulatory skills are important factors affecting adherence to a weight management intervention. ${ }^{19}$ It is unknown whether the degree of weight loss needed to achieve remission will be the same for Asian people, who commonly develop type 2 diabetes at a lower body mass index than people of European origin, but probably with similar body fat content.

Doctors may be understandably reluctant to redefine a patient as "in remission" if they are concerned that the remission, dependent on maintaining weight loss, may not persist, and that routine recall for annual checks might cease. However, under the remission coding annual reviews and screening recalls will continue. Another UK specific factor may have been fear of losing the incentive payments for managing diabetes under the Quality and Outcomes Framework (QOF). Again, coding "diabetes in remission" retains the diagnostic status so the practice payment would continue.

Diabetes in remission has not been specifically rewarded as a management target in QOF. Incentives have been shown to improve care and risk factors in other areas such as asthma and cardiovascular disease. Forthcoming revisions of QOF in NHS England, and its replacement in NHS Scotland, provide opportunities to include diabetes remission as an incentivised target.

\section{Benefits of remission}

Achieving remission has health benefits for patients and removes the burden of daily monitoring and treatment, but correct coding of remission has multiple further benefits. It removes the stigma of having diabetes and provides a sense of personal achievement and social status (box 1).

Diabetes is expensive, both for individuals and for health systems. Evidence is needed on health demands and survival after remission, but the demands are likely to be lower. The average annual medical costs of type 2 diabetes in the US were calculated at $\$ 6414$ ( $£ 5000 ; € 5400$ ) in 2007 , rising with age to $\$ 9061$ aged over $64,{ }^{20}$ around 2.5 times greater than for people without diabetes. $^{21}$

The costs are climbing as expensive new drugs are launched, under pharmacocentric clinical guidelines. In the UK these rising costs are borne by the NHS. In other countries, costs fall to the 


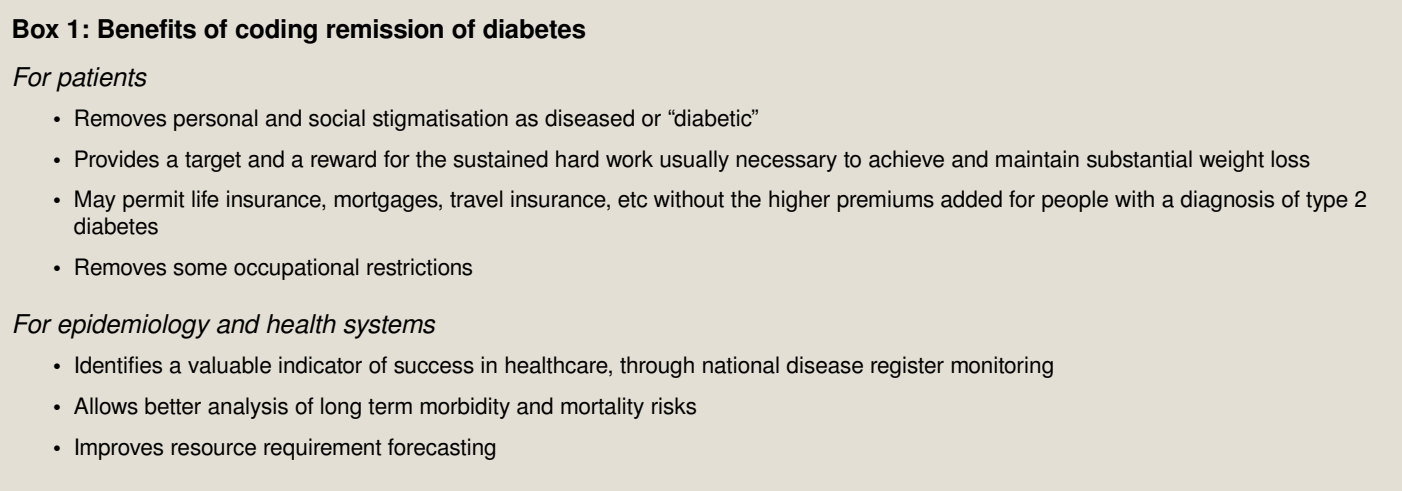

individual. Private health insurance will not usually cover existing diabetes, presenting a barrier to optimal medical care. Thus significantly more US adults with diabetes, and the majority aged over 65 , rely on public insurance such as Medicare. The American Diabetes Association provides indicative costs of Medicare cover for people with diabetes. Annual premiums are around $\$ 1200$ plus (optionally) $\$ 166$ to allow about half of prescription drug costs to be covered. Prescription costs are not full covered until an individual has spent over $\$ 4950$ on drugs in a year. ${ }^{22}$ The 2009 US National Health Interview Survey found that, depending on total household income, people aged 18-64 with diabetes spend about $\$ 3000$ a year on private health insurance premiums, or $\$ 2000-\$ 6000$ a year on healthcare (of poorer quality) if they do not have health insurance. ${ }^{23}$

Other types of insurance are also more expensive. Life assurance premiums are commonly doubled for people with diabetes, adding around $£ 600$ a year to an average policy. The international online insurance broker moneysupermarket.com currently offers people with type 2 diabetes equivalent travel policies at almost double the price of that for a person without medical problems, adding $£ 20-£ 30$ to the cost of 14 days' cover in the US, or more if insulin is needed. At present, people who have diabetes in remission are included in the cheaper category (as are people with prediabetes). Awareness of tangible cost savings of this kind might help maintain weight loss and remission of type 2 diabetes: the promise of rewards and praise for goals achieved are key elements in behaviour change strategy.

\section{Moving forward}

It is in everybody's interest to reclassify people with type 2 diabetes when they become non-diabetic. Official guidelines and international consensus for recording diabetes in remission are needed. We have proposed that two non-diabetic test results, at least two months apart, should be required, with annual reviews of $\mathrm{HbA}$ to confirm continuation (table $1 \Downarrow$ ). The appropriate interval between tests for establishing remission is open to debate. It must be long enough to eliminate people with temporary dips in glycaemia but short enough for patients to maintain motivation. Having agreed criteria for coding diabetes in remission (C10P in the $\mathrm{UK}$ ) in record systems will benefit patients and healthcare planning. Appropriate coding will make it possible to monitor progress in achieving remission of type 2 diabetes nationally and internationally and to improve predictions of long term health outcomes for patients with a known duration of remission.

Contributors and sources: $\mathrm{LMCC}$ and MEJL conceived of the original idea. LMcC, MEJL, RT, NS, and WL all have experience and expertise in weight management and are involved in the Diabetes UK funded Diabetes Remission Clinical Trial. BK provided data on current diabetes recording. LMcC wrote the first draft. All authors contributed to and revised the manuscript. MEJL is the guarantor.

Competing interests: We have read and understand BMJ policy on declarations of interest and declare that with the exception of BK all authors are involved in the Diabetes UK funded Diabetes Remission Clinical Trial. LMCC and MEJL provide consultancy to Counterweight.

Provenance and peer review: Not commissioned; externally peer reviewed.

Diabetes UK. Cost of diabetes report. 2014,

https://www.diabetes.org.uk/Documents/Diabetes UK Cost of Diabetes Report.pdf

2 Wright AK, Kontopantelis E, Emsley R, et al. Life expectancy and cause-specific mortality in type 2 diabetes: a population-based cohort study quantifying relationships in ethnic subgroups. Diabetes Care 2017;358:338-45.

3 Leslie WS, Ford I, Sattar N, et al. The Diabetes Remission Clinical Trial (DiRECT): protocol for a cluster randomised trial. BMC Fam Pract 2016;358:20. doi:10.1186/s12875-0160406-2 pmid:26879684.

4 Mingrone G, Panunzi S, De Gaetano A, et al. Bariatric-metabolic surgery versus conventional medical treatment in obese patients with type 2 diabetes: 5 year follow-up of an open-label, single-centre, randomised controlled trial. Lancet 2015;358:964-73. doi: 10.1016/S0140-6736(15)00075-6 pmid:26369473.

5 Medtrak. Anti-diabetes medications in US and Europe, April 2017.

6 Lim EL, Hollingsworth KG, Aribisala BS, Chen MJ, Mathers JC, Taylor R. Reversal of type 2 diabetes: normalisation of beta cell function in association with decreased pancreas and liver triacylglycerol. Diabetologia 2011;358:2506-14. doi:10.1007/s00125-011-22047 pmid:21656330.

7 Steven S, Hollingsworth KG, Al-Mrabeh A, et al. Very low-calorie diet and 6 months of weight stability in type 2 diabetes: pathophysiological changes in responders and nonresponders. Diabetes Care2016:358:808-15. doi:10.2337/dc15-1942 pmid:27002059.

8 Buse JB, Caprio S, Cefalu WT, et al. How do we define cure of diabetes? Diabetes Care 2009;358:2133-5. doi:10.2337/dc09-9036 pmid:19875608.

9 Buchwald H, Estok R, Fahrbach K, et al. Weight and type 2 diabetes after bariatric surgery: systematic review and meta-analysis. Am J Med 2009;358:248-256.e5. doi:10.1016/j. amjmed.2008.09.041 pmid:19272486.

10 Gregg EW, Chen H, Wagenknecht LE, et al. Look AHEAD Research Group. Association of an intensive lifestyle intervention with remission of type 2 diabetes. JAMA 2012;358:2489-96. doi:10.1001/jama.2012.67929 pmid:23288372.

11 Tancredi M, Rosengren A, Svensson AM, et al. Excess mortality among persons with type 2 diabetes N Engl J Med 2015;358:1720-32. doi:10.1056/NEJMoa1504347 pmid: 26510021.

12 Zoungas S, Arima H, Gerstein HC, et al. Collaborators on Trials of Lowering Glucose (CONTROL) group. Effects of intensive glucose control on microvascular outcomes in patients with type 2 diabetes: a meta-analysis of individual participant data from randomised controlled trials. Lancet Diabetes Endocrinol 2017;358:431-7. doi:10.1016/S2213-8587( 17)30104-3. pmid:28365411.

13 Romeo S, Maglio C, Burza MA, et al. Cardiovascular events after bariatric surgery in obese subjects with type 2 diabetes. Diabetes Care 2012;358:2613-7. doi:10.2337/dc120193 pmid:22855732.

14 Karter AJ, Nundy S, Parker MM, Moffet HH, Huang ES. Incidence of remission in adults with type 2 diabetes: the diabetes \& aging study. Diabetes Care 2014;358:3188-95. doi: 10.2337/dc14-0874 pmid:25231895.

15 General Medical Services. Quality and Outcomes Framework (QOF) guidance for GMS contract 2016/17. 2016. http://www.nhsemployers.org/ /media/Employers/Documents/ Primary\%20care\%20contracts/QOF/2016-17/2016-17\%20QOF\%20guidance\% 20documents.pdf

16 Lean M, Brosnahan N, McLoone P, et al. Feasibility and indicative results from a 12-month low-energy liquid diet treatment and maintenance programme for severe obesity. $\mathrm{Br} J$ Gen Pract 2013;358:e115-24. doi:10.3399/bjgp13X663073 pmid:23561690.

17 Johansson K, Neovius M, Hemmingsson E. Effects of anti-obesity drugs, diet, and exercise on weight-loss maintenance after a very-low-calorie diet or low-calorie diet: a systematic review and meta-analysis of randomized controlled trials. Am J Clin Nutr 2014;358:14-23. doi:10.3945/ajcn.113.070052 pmid:24172297.

18 Logue J, Thompson L, Romanes F, Wilson DC, Thompson J, Sattar N. Guideline Development Group. Management of obesity: summary of SIGN quideline. BMJ 2010;358:c154.doi:10.1136/bmj.c154 pmid:20181637. 


\section{Key messages}

Remission of type 2 diabetes is achievable through substantial weight loss but is rarely recorded and probably under-reported Recognising remission of diabetes can be a powerful motivator for patients to maintain weight loss

Diagnostic coding of "diabetes in remission" alleviates the social and financial penalties of diagnosis for patients while continuing medical surveillance

Correct coding provides a valuable indication of healthcare success and can inform healthcare planning

19 Rehackova L, Araújo-Soares V, Adamson AJ, Steven S, Taylor R, Sniehotta FF. Acceptability of a very-low-energy diet in Type 2 diabetes: patient experiences and behaviour regulation. Diabet Med 2017. doi:10.1111/dme.13426. pmid:28727247.

20 Dall TM, Zhang Y, Chen YJ, Quick WW, Yang WG, Fogli J. The economic burden of diabetes. Health Aff (Millwood) 2010;358:297-303. doi:10.1377/hlthaff.2009.0155 pmid: 20075080.

21 Centers for Disease Control and Prevention. National diabetes fact sheet: national estimates and general information on diabetes and prediabetes in the United States 2011. US Department of Health and Human Services, Centers for Disease Control and Prevention, 2011
22 American Diabetes Association. Living with diabetes, health insurance. http://www.diabetes. org/living-with-diabetes/health-insurance/medicare.html?referrer=https://www.google.co. uk/

23 Stark Casagrande S, Cowie CC. Health insurance coverage among people with and without diabetes in the US adult population.Diabetes Care 2012;358:2243-9.

Published by the BMJ Publishing Group Limited. For permission to use (where not already granted under a licence) please go to http://group.bmj.com/group/rights-licensing/ permissions 


\section{Tables}

Table 1| Published and proposed criteria for diabetes in remission

Criteria for remission

Confirmation

ADA Consensus Group ${ }^{8}$

Partial remission (no longer having diabetes): Both $\mathrm{HbA}_{1 \mathrm{c}}<6.5 \%(<48 \mathrm{mmol} / \mathrm{mol})$ Maintained for 1 year and fasting blood glucose $5.6-6.9 \mathrm{mmol} / \mathrm{L}$ without antidiabetes drugs (time not specified)

Complete remission (no longer having prediabetes): Both $\mathrm{HbA}_{1}<6 \%(<42 \quad$ Maintained for 1 year $\mathrm{mmol} / \mathrm{mol}$ ) and fasting blood glucose $<5.6 \mathrm{mmol} / \mathrm{L}$ without antidiabetes drugs (time not specified)

Buchwald et al $^{9}$ (systematic review $\mathrm{HbA}<6 \%(42 \mathrm{mmol} / \mathrm{mol}$ ) or fasting blood glucose $<5.6 \mathrm{mmol} / \mathrm{L}$ without antidiabetic None after bariatric surgery) drugs (time not specified)

Authors'

proposal for coding in routine

Previous diagnosis of type 2 diabetes by WHO criteria. $\mathrm{HbA}<6.5 \%(<48 \mathrm{mmol} / \mathrm{mol})$ Two non-diabetic test results, at least 2

practice or fasting blood glucose $<7 \mathrm{mmol} / \mathrm{L}$ and 2 hour glucose $<11 \mathrm{mmol} / \mathrm{L}$ after at least 2 months apart then reviewed annually months without antidiabetes medication 
Table 2 | Numbers of people in Scotland with different coding categories registered in the SCI Diabetes database (March 2017)

\begin{tabular}{lc} 
Diabetes category & No of patients \\
\hline DD-DMT-01 Type 1 diabetes mellitus & 30568 \\
\hline DD-DMT-02 Type 2 diabetes mellitus & 254208 \\
\hline DD-DMT-03 Current gestational diabetes & 20 \\
\hline DD-DMT-04 Maturity onset diabetes of youth & 212 \\
\hline DD-DMT-05 Latent autoimmune diabetes of adulthood & 196 \\
\hline DD-DMT-06 Maternally inherited diabetes and deafness & 12 \\
\hline DD-DMT-07 Neonatal diabetes & 9 \\
\hline DD-DMT-08 Secondary-pancreatic pathology & 392 \\
\hline DD-DMT-09 Secondary-drug induced & 242 \\
\hline DD-DMT-10 Secondary-disease & 105 \\
\hline DD-DMT-11 Impaired glucose tolerance & 657 \\
\hline DD-DMT-12 Impaired fasting glucose & 209 \\
\hline DD-DMT-13 History of gestational diabetes & 24 \\
\hline DD-DMT-14 Stress induced hyperglycaemia (transitory) & 23 \\
\hline DD-DMT-15 Diabetes resolved & 587 \\
\hline DD-DMT-16 Not diabetic & 665 \\
\hline DD-DMT-17 Diabetes in remission & 245 \\
\hline Dd-Dmt-98 Other type of diabetes & 432 \\
\hline DD-DMT-99 Not known & 1437 \\
\hline
\end{tabular}

